\title{
La toma militar en Siloé: memoria colectiva e identidad política.
}

Eliana Donneys Bastidas ${ }^{1} \quad \begin{gathered}\text { Sara Natalia Pérez } \\ \text { elianadonneys@outlook.com } \\ \text { snatalia94@hotmail.com }\end{gathered}$

Artículo de investigación recibido el 15/02/2018

y aprobado el 02/03/2019

Cómo citar este artículo:

Donneys Bastidas, E., \& Pérez, S. N. (2020). La toma militar en Siloé: memoria colectiva e identidad política. TransPasando Fronteras, (15). https://doi.org/10.18046/retf. i15.2758

${ }^{1}$ Estudiante de Ciencia Política con énfasis en RRII y Antropología, Universidad Icesi. Correo electrónico: eliana.donneys@correo.icesi.edu.co

${ }^{2}$ Estudiante de Psicología y Sociología, Universidad Icesi. 


\section{Resumen}

El presente trabajo tiene por objetivo analizar el grado de importancia subjetiva que los habitantes de los barrios Lleras Camargo, Pueblo Joven y Tierra Blanca, le otorgan a la toma militar llevada a cabo el 1 de diciembre de 1986, en Siloé, Cali, Valle del Cauca. La cuestión a considerar es cómo y en qué grado dicho hecho se interpreta en la memoria colectiva de la población de la comuna 20 y se cristaliza en las esferas, tanto personales como colectivas, y en qué medida esto se vincula con las condiciones sociodemográficas como la edad o rango generacional y la identidad política de los encuestados. Para esto se ahonda en cuatro dimensiones analíticas relevantes: la experiencia subjetiva de la toma militar (la importancia de este hecho, el conocimiento del mismo y las emociones que suscitó); la percepción de la toma militar (el grado de violencia, la justificación, el apoyo social y la resistencia que tuvo la misma); la percepción acerca de las causas de la toma militar (rol de los actores involucrados en el hecho, responsabilidad de estos en las acciones que se llevaron a cabo) y la proyección futura (cuestionamientos contrafactuales y enseñanzas de la toma militar), cruzando las mismas con la variable de edad -o rango generacional- e identidad política (Manzi, Helsper, Ruiz, Krause \& Kronmüller, 2003).

Palabras clave: Siloé; toma militar 1985; identidad política; memoria colectiva; rango generacional. 


\title{
The military takeover in Siloé: collective memory and political identity.
}

\begin{abstract}
The main objective of this paper is to analyze the degree of the subjective importance that the inhabitants of the Lleras Camargo, Pueblo Joven and Tierra Blanca neighborhoods grant to the military takeover carried out on December 1 of 1986, in Siloé, Cali, Valle del Cauca. The question to be considered is how and to what degree this event is interpreted in the collective memory of the commune 20 population. Additionally, how it materializes in the population's personal and collective spheres, and to what extent this interpretation is linked to sociodemographic conditions such as age or generational range and the political identity of the respondents. In order to accomplish this analysis, we will delve into four relevant analytical dimensions: the subjective experience of the military takeover (the importance of this event, the knowledge of it and the emotions it aroused); the perception of the military takeover (the degree of violence, justification, social support and the resistance that came with it); the perception about the causes of the military takeover (role of the actors involved in the event, responsibility of these in the actions that were carried out) and the future projection (counterfactual questions and lessons learned from the military takeover), crossing the same with the variable of age -or generational rank- and political identity (Manzi et al., 2003).
\end{abstract}

Keywords: Siloé; military takeover 1985; political identity; collective memory; generational range. 
"La vida no es la que uno vivió, sino la que uno recuerda y cómo la recuerda para contarla"

(García, 2002, p.2)

Siloé es el nombre popular por el cual se le conoce a la comuna 20 de la ciudad de Cali, compuesta por 8 barrios y tres Urbanizaciones. Se encuentra ubicada en la parte occidental de la metrópoli en el piedemonte de la cordillera Occidental entre los Farallones de Cali, la Avenida De Los Cerros, el Cerro Cristo Rey y el Cerro Bataclán. El poblamiento en Siloé se ha desarrollado en un $80 \%$ por procesos ilegales de urbanización: invasión o urbanización clandestina; mientras que el $20 \%$ restante se ha desarrollado dentro de los procesos legales establecidos (Caldono, Echeverry \& Pacheco, 2012).

El nombre de Siloé fue escogido en honor al primer barrio que fue fundado en este sector, en la época en donde la mayor parte de su territorio estaba constituido por flora y fauna, con presencia de una mina carbonífera. Con el paso del tiempo, a medida que las construcciones fueron avanzando y se presentaron mejores servicios públicos, se reconoció a los barrios la comuna 20, no como extensiones sub-urbanas, sino como barrios propiamente constituidos. No obstante, a lo largo del recorrido histórico de Santiago de Cali, Siloé ha estado asociada con altos índices de violencia, lo cual ha creado un imaginario en los ciudadanos caleños que tiende a estigmatizar a los habitantes de este sector, aunque al mismo tiempo, ha incentivado la creación de iniciativas -tanto en el ámbito público como privado- que buscan ayudar a reconstruir el tejido social y potencializar procesos sociales, culturales, económicos, medio ambientales y de recuperación urbana de la comuna (Caldono, Echeverry \& Pacheco, 2012).

Ahora bien, es importante señalar que la toma militar llevada a cabo el 1 de diciembre de 1986 en Siloé fue, a grandes rasgos, una línea 
de acción del ejército que respondía a las jornadas sistemáticas de 'limpieza social' de la época, y fue coadyuvada por la policía nacional; por medio de la operación denominada "Cali, Navidad Limpia" o "Cali linda en navidad" (Ulloa, 2011), se buscó mermar o erradicar la presencia de militantes del M-19 en la Comuna 20 de Cali. Por tanto, la problemática central que se pretende dilucidar en el presente escrito, radica en analizar el grado de importancia subjetiva que la población de Siloé, más puntualmente, los habitantes de los barrios Lleras Camargo, Pueblo Joven y Tierra Blanca, le asignan a los acontecimientos ocurridos en la Toma Militar el 1 de Diciembre de 1985, en la cual, estuvieron involucrados actores estatales como el ejército nacional y la policía, actores al margen de la ley como los militantes del grupo guerrillero M-19, y actores pertenecientes a la población civil como los residentes de las zonas mencionadas.

La cuestión a considerar es, por medio de la conjunción entre la teoría presentada y la indagación estadística, cómo y en qué grado este hecho se interpreta en la memoria colectiva de los habitantes de los barrios y se cristaliza en las esferas, tanto personales como colectivas y en qué medida esto se vincula con las condiciones sociodemográficas como la edad o rango generacional y la identidad política de los encuestados, cabe aclarar que la variable de identidad política hace referencia a la afinidad de cada encuestado dentro del espectro ideológico colombiano, comprendido entre partidos de izquierda, partidos de centro y partidos de derecha. La variable identidad política es de vital importancia ya que permite el contraste de valores cuantitativos hallados en el presente estudio con los planteamientos de la teoría de memoria colectiva de Halbwach según los cuales el factor social incide no sólo en la creación de los recuerdos sino también en su futura evocación e interpretación.

Colorario a lo anterior, se ahonda en cuatro dimensiones analíticas relevantes: la experiencia subjetiva de la toma militar (la importancia de este hecho, el conocimiento del mismo y las emociones que suscitó); la percepción de la toma militar (el grado de violencia, la justificación, el 
apoyo social y la resistencia que tuvo la misma); la percepción acerca de las causas de la toma militar (rol de los actores involucrados en el hecho, responsabilidad de estos en las acciones que se llevaron a cabo) y la proyección futura (cuestionamientos contra-factuales y enseñanzas de la toma militar), cruzando las mismas con la variable de edad -o rango generacional- e identidad política ${ }^{3}$.

La presente investigación busca comprender un hecho social específico de violencia, que hace parte de un contexto urbano. Para ello, se pretende construir análisis y explicaciones más integrales a partir de teorías que pertenecen a dos ciencias diferentes como lo son la Ciencia Política y la Psicología. Así, por medio de este enfoque interdisciplinar, se procura, no sólo aplicar teorías sociales aprendidas a lo largo de nuestras carreras, sino también crear nuevo conocimiento, a partir de lo que el análisis conjunto de dos disciplinas sobre un hecho social permite lograr, pues el enfoque interdisciplinar permite una mejor comprensión de los fenómenos sociales, a la vez que, abre la puerta a un abanico amplio de explicaciones y soluciones a problemáticas sociales (García, 2013).

\section{Memoria colectiva}

Siguiendo los postulados del filósofo y sociólogo Maurice Halbwachs (2002), el término memoria colectiva puede ser definido como la memoria de un grupo de individuos que reconstruye su pasado a partir de referentes de su presente. Halbwachs asegura que la memoria continuamente tiene un componente social, pues los recuerdos se construyen a partir de experiencias que ocurren en relación con otras

3 Los resultados expuestos a continuación corresponden al trabajo de recolección de datos desarrollado durante el semestre 2015-1 en la clase taller de investigaciónSondeo, por los estudiantes de la misma cohorte de la Universidad Icesi. 
personas; es así como lugares, palabras, ideas, imaginarios elaborados y transmitidos por otros, por medio de la comunicación interpersonal, juegan un papel significativo en la memoria. Colorario a lo anterior, la memoria colectiva no es estable sino que se encuentra siempre en constante cambio como una recreación del pasado, debido a su carácter constructivo antes de ser meramente reproductiva. De este modo, el factor social de los recuerdos, para Halbwachs, no sólo se evidencia en la fabricación de los mismos, sino también en la influencia que la sociedad tiene en su interpretación y posterior evocación (Halbwachs, 2002).

Una sociedad elige honrar la memoria de personajes o momentos significativos por medio de monumentos, esculturas, pinturas, textos o cualquier otra expresión artística que permita materializar los recuerdos. Pero de igual forma, se elige qué eventos no recordar, o de alguna manera no reconstruir lo acontecido a partir de una historia que no es fiel a lo que realmente ocurrió (Halbwachs, 2002). Por este motivo, la memoria colectiva no es una copia exacta de la realidad de una sociedad, es decir, puede que no represente con exactitud los detalles de un suceso, pero sí da luces para analizar los factores particulares que incidieron en la subjetividad de los individuos que vivieron un hecho determinado y crearon una nueva verdad. A esto vale agregar que, debido a que la memoria colectiva es compartida, transmitida y construida en grupo, la comunicación desempeña un papel fundamental en su constitución. Así pues, la tradición oral, los medios masivos de comunicación, la opinión pública, el internet, entre otros, son fenómenos que influyen en la creación de las memorias y su perpetuidad (Páez, Nekane y González, 1998).

De esta manera, la naturaleza social y grupal de lo vivido trae como consecuencia que los sucesos evocados frecuentemente no coincidan en una sola memoria, sino en una pluralidad de ellas, muchas veces contrapuestas. En el caso del barrio Siloé esto se puede evidenciar, pues entre las personas encuestadas, las cuales se encuentran en 
distintas categorías de edad o grupo generacional, se observan distintas percepciones sobre lo ocurrido en la toma militar. En este punto es menester traer a colación la categoría de rango generacional, ya que la misma va estrechamente ligada a la cuestión sobre memoria colectiva, siguiendo algunos estudios sobre socialización política, Manzi et al (2003) aseguran al respecto:

Especialmente la teoría de "ciclos de vida", que coinciden en definir a la etapa de la adolescencia tardía o de la adultez temprana como el período más decisivo para perfilar opiniones, actitudes y orientaciones en torno a lo político (Steward y Healy, 1989; Sears, 1990). Esta etapa en la vida no es importante solamente para formar actitudes u orientaciones políticas, también es el período en el cual las personas muestran mayor acuerdo con comportamientos políticos más extremos y desviados (Watts, 1999). Estos fenómenos indican que la adolescencia es un período importante para la formación de una identidad política que sigue vigente en etapas posteriores. La significación subjetiva de los hechos históricos que afectan a distintas cohortes etarias también ha sido empleada para comprender la emergencia de distintas generaciones (p.180).

Por ello, en el presente escrito se optó por dividir a la población en siete grupos etarios denominados rangos generacionales, en aras de evidenciar las similitudes y diferencias entre los mismos y sus respuestas.

\section{Metodología}

El instrumento empleado para recolectar los datos fue una encuesta elaborada por los estudiantes de la Universidad Icesi en el semestre 2015-1, que constaba de ochenta y seis preguntas, las cuales ahondaron en las cuatro dimensiones descritas anteriormente. La metodología de muestreo aplicada fue bola de nieve, así pues, se recolectaron ciento setenta y cinco encuestas en los barrios Lleras Camargo, Pueblo Joven y Tierra Blanca, las cuales se depuraron y sistematizaron, siendo el insumo principal del presente análisis. 


\section{Muestra}

Ahora bien, al examinar la comuna 20 de Cali, se puede observar que está compuesta por ocho barrios y tres urbanizaciones como son: Belén, Belisario Caicedo, Brisas de Mayo, Cementerio Carabineros, El Cortijo, Lleras Camargo, Pueblo Joven, La Sultana, Siloé, Tierra Blanca, Venezuela Urbanización Cañaveralejo, de los cuales un $80 \%$ se desarrollaron por procesos ilegales de urbanización, como invasiones o urbanizaciones clandestinas. Según el censo del 2005 del DANE se completa un número de 65.440 habitantes de estrato 1 y 2 en este sector" (Desde abajo, 2011), además, "las características étnicas de su población se clasifican entre afrocolombianos, mestizos e indígenas (Betancourt \& Molina Orrego, 2014, p.41).

Siloé se ubica en la Comuna 20, en el sector occidental de la ciudad de Cali, Valle del Cauca. Esta comuna alberga el 3,1\% del total de viviendas de la metrópoli (Alonso, Solano, Vera, \& Gallego, 2007) y en ella habita el 3,2\% del total de habitantes de Santiago de Cali, de los cuales, más de la mitad son mujeres con un $51,5 \%$ y el $48,5 \%$ son hombres. En lo que respecta a la población encuestada, la cual alcanza un total 175 personas ( $N=175)$, el 38,3\% de estas residía en Tierra Blanca, el 28,6\% en Pueblo Joven, y el 25,7\% en Lleras Camargo, dando como total un $92,6 \%$ de encuestados moradores de los barrios de interés del presente estudio.

De las 175 personas encuestadas, el 70,3\% fueron mujeres y el $29,7 \%$ restante hombres. Las edades del total de personas encuestadas fueron agrupadas en siete rangos generacionales: $17,7 \%$ del total de los encuestados tenía entre 18 a 24 años, entre 25 a 31 años se ubicaba un $13,1 \%$ de encuestados, entre 32 y 38 años se ubicaba un 13,7\% de encuestados, entre 39 a 45 años se ubicaba un 12\% de encuestados, entre 46 a 52 años se ubicaba un 13,7\% de encuestados, entre 53 a 59 años se ubicaba un 13,1\% de encuestados, y el 14,9\% de los encuestados afirmó tener más de 60 años, por lo tanto, al encontrar 
representatividad equilibrada en la edad o rango generacional, y a la vez, gracias a la relación directa que esta variable tiene con la memoria colectiva, se empleó como el ítem sociodemográfico principal para ser cruzado con los resultados de las preguntas que se expondrán a lo largo del trabajo.

En lo que respecta al promedio salarial del total de los encuestados, este oscilaba entre 200.001 y 400.000 pesos, aunque el dato que más se repitió como respuesta fue de 0 a 200.000 pesos. La desviación estándar arroja que los encuestados pueden desviarse del promedio en una categoría, y la mediana muestra que el 50\% de las personas encuestadas ganaban hasta la categoría de salario que abarca entre 200.001 y 400.000 pesos. El promedio del máximo nivel educativo alcanzado por las personas encuestadas fue secundaria incompleta, aunque el dato que más se repitió fue secundaria completa. La desviación estándar del máximo nivel educativo alcanzado arrojó que los encuestados podían desviarse del promedio dos casillas aproximadamente, y la mediana expuso que el 50\% del total de los encuestados habían alcanzado como máximo nivel educativo hasta secundaria incompleta.

En cuanto a identidad política se refiere, inscrita en determinado partido político, más de la mitad del total de encuestados, es decir, un $63 \%$ decía ser apolítico, mientras que un 10,9\% de derecha y un 15,4\% de izquierda, el 4\% afirmaba ser de Centro, y un 2,3\% perteneciente a otra denominación en el espectro político (esta podría ser un matiz entre las nombradas anteriormente como: centro-derecha, centroizquierda) 


\section{Presentación y discusión de resultados}

\section{Dimensión No. 1: experiencia subjetiva del hecho}

A la pregunta de única respuesta sobre la importancia de la toma, del total de personas que respondieron que para ellos había tenido mucha importanciaestesuceso:el 25,6\% tenía de 60 años enadelanteyel 23,1\% entre 53 y 59 años; más de la mitad, es decir, 53,8\% se denominaron apolíticos. Del total de personas que respondieron que para ellos había tenido poca importancia el suceso de la toma, el 29,4\% tenía entre 39 a 45 años, el 23,5\% entre 53 a 59 años y el 17,6\% más de 60 años en adelante; además, el 47,1\% se denominaban apolíticos, mientras que el $29,4 \%$ de izquierda. Del total de personas que respondieron que para ellos este suceso no tenía ninguna importancia, el 20,6\% tenía entre 39 a 45 años y el 17,6\% entre 18 a 24 años; la mayoría, es decir un 73,5\% se denominaban apolíticos. De las personas que contestaron "No sabe/ No responde" respecto a la importancia de la toma, el 26,7\% tenía de 46 a 52 años, el 26,7\% de 53 a 59 años y el 20\% 60 años en adelante (Ver Gráfico No 1 y Gráfico No. 2).

En este caso se puede evidenciar que los hechos ocurridos en la toma fueron considerados importantes y marcaron la memoria social o colectiva (Halbwachs, 2002) principalmente de aquellas personas cuya edad en el momento que ocurrieron los hechos oscilaba desde los 20 años en adelante. De igual forma, se observa que los rangos de edad de aquellos que aseguraron que la toma militar no había tenido ninguna importancia están entre los 10 y los 15 años o no habían nacido en el momento de los hechos. Se puede demostrar así, cómo la memoria colectiva de los habitantes del barrio se encuentra marcada especialmente por las experiencias vivenciadas directamente en relación con la toma, más que por los recuentos de los hechos o la transmisión del impacto de lo sucedido a través de cualquier medio de comunicación, oral o escrito a las nuevas generaciones (Halbwachs, 2002). A la vez, en contraposición, cabe destacar el porcentaje 
significativo de apolíticos en los tres últimos rangos generacionales que manifestaron no saber o no responder sobre la importancia de la toma (Ver Gráfico No 1 y Gráfico No. 2).

Gráfico No. 1: ¿Qué tan importante es para usted dicha Toma militar?

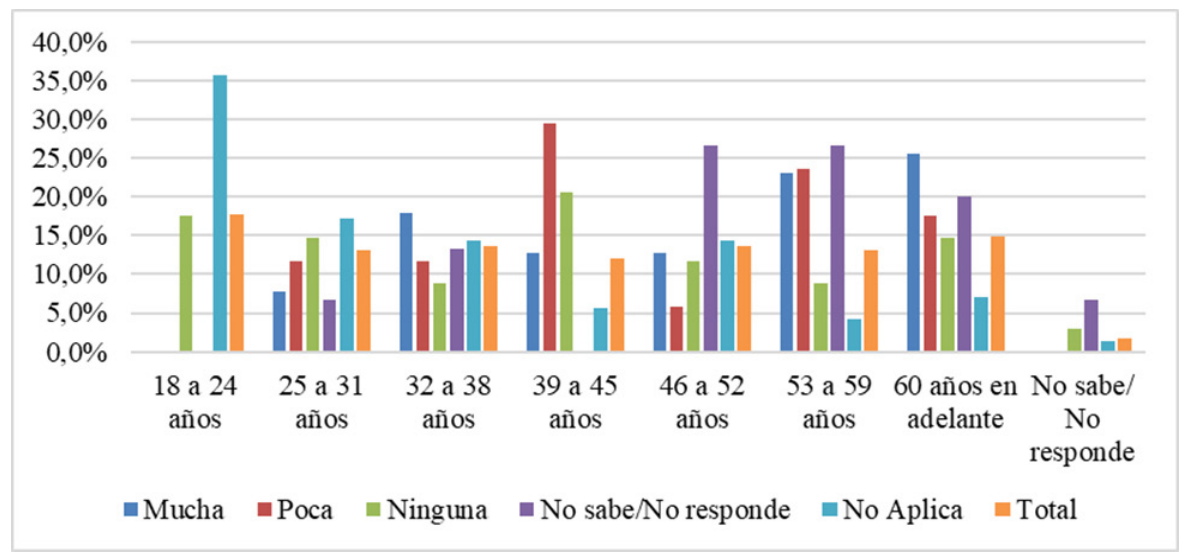

Fuente: Encuesta realizada para el curso Taller de Investigación-Sondeo

(primer semestre académico de 2015). Elaboración propia.

Gráfico No. 2: ¿Qué tan importante es para usted dicha Toma militar?

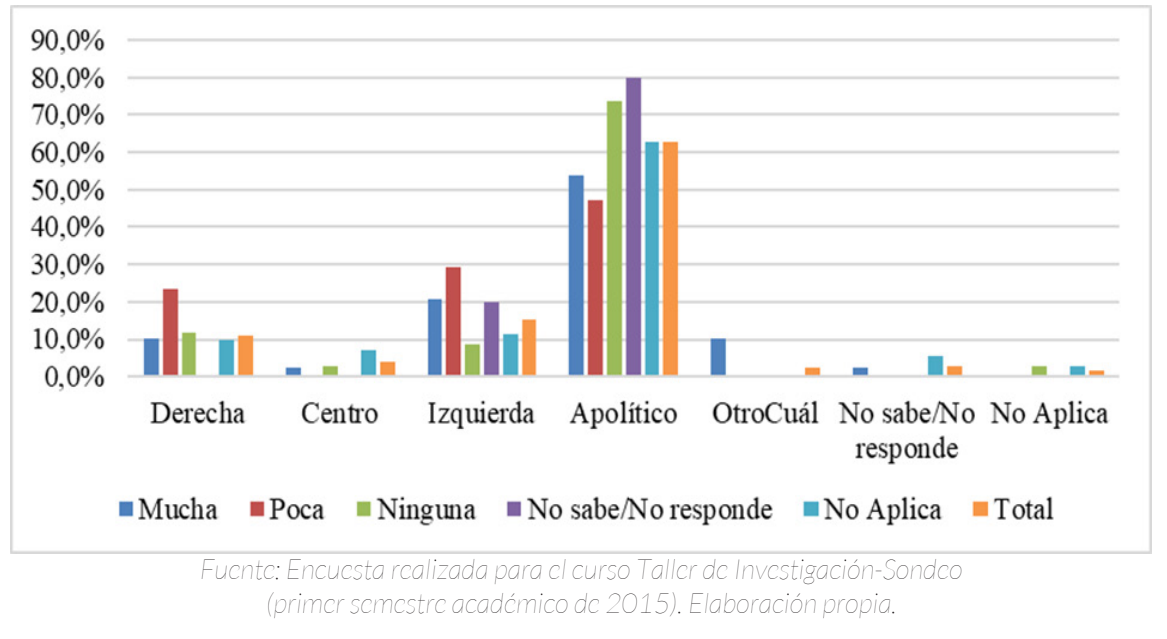

${ }^{4}$ Las personas encuestadas que su respuesta corresponde a No Aplica, no conocen el hecho de la toma militar en Siloé. 
A la pregunta de múltiple respuesta sobre qué emociones tiene cuando piensa en el suceso de la toma militar, del total de personas que respondieron que sentían pena, el 33,3\% se ubicaba en el rango de edad entre 32 a 38 años y el 22,2\% entre 39 a 45 años; la mayoría, con un $66,7 \%$ se denominaban apolíticos. Del total de personas que respondieron que sentían impotencia, el 27,3\% se ubicaba entre los 53 a los 59 años, el 22,7\% entre los 46 a los 52 años y el 18,2\% de 60 años en adelante; el 45,5\% se denominaban como apolíticos y el 36,4\% eran de un partido de izquierda. Del total de personas que respondieron que sentían indiferencia, el 25\% tenía entre 39 a 45 años, el 16,7\% entre 18 a 24 años y el 16,7\% más de 60 años en adelante; la mayoría con un 79,2\% se denominaban apolíticos. Del total de personas que respondieron que sentían rabia, el 29\% tenían entre 53 a 59 años y el 19,4\% de 60 años en adelante; más de la mitad, es decir el 61,3\% se denominaban como apolíticos. Del total de personas que respondieron que sentían alegría en cuanto a la edad, la población se dividió en cuatro categorías, cada una equivalente a un 25\%, entre 32 a 38 años, entre 39 a 45 años, entre 46 a 52 años y entre 53 a 59 años edad; un 50\% se denominaba apolítico, el $25 \%$ perteneciente a un partido de izquierda y el 25\% a un partido de derecha. Del total de personas que respondieron que sentían orgullo, la mitad con un 50\% tenían entre 32 a 38 años; la mitad con un 50\% se denominaba apolítico, el 25\% perteneciente a un partido de izquierda y el 25\% a un partido de derecha (Ver Gráfico No. 3 y Gráfico No. 4)

Colorario al análisis de las dos respuestas de la dimensión No. 1 expuestas anteriormente, cabe señalar que la Toma Militar fue un evento de importancia subjetiva para los habitantes del barrio encuestados cuyas edades en el momento de aplicar la misma se encontraban desde los 46 años en adelante, debido a que ellos fueron quienes vivenciaron los sucesos en una edad adulta -cuando ocurrieron los hechos tenían 14 años o más- y sus posteriores consecuencias. Lo anterior se evidencia en que este hecho suscitó sentimientos intensos 
tanto negativos (rabia, impotencia) como positivos (alegría, orgullo) en este rango generacional, mientras que el sentimiento de indiferencia cuenta con las cifras más bajas desde los 46 años en adelante, en comparación con las demás emociones. No obstante, a pesar de tener una alta significancia en la esfera personal (sentimientos) también se puede observar que en la esfera política o identidad política (partido político) estos contrastan con una definición de neutralidad significativa (apolítico) bastante marcada.

Gráfico No. 3: ¿Qué emociones siente al hablar de la Toma militar?

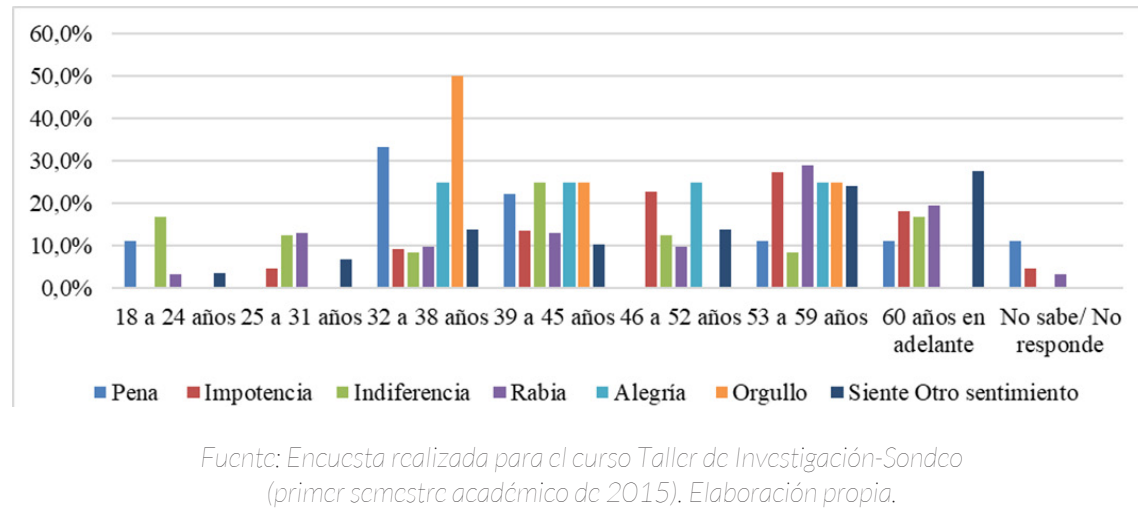

Gráfico No.4: ¿Qué emociones siente al hablar de la Toma militar?

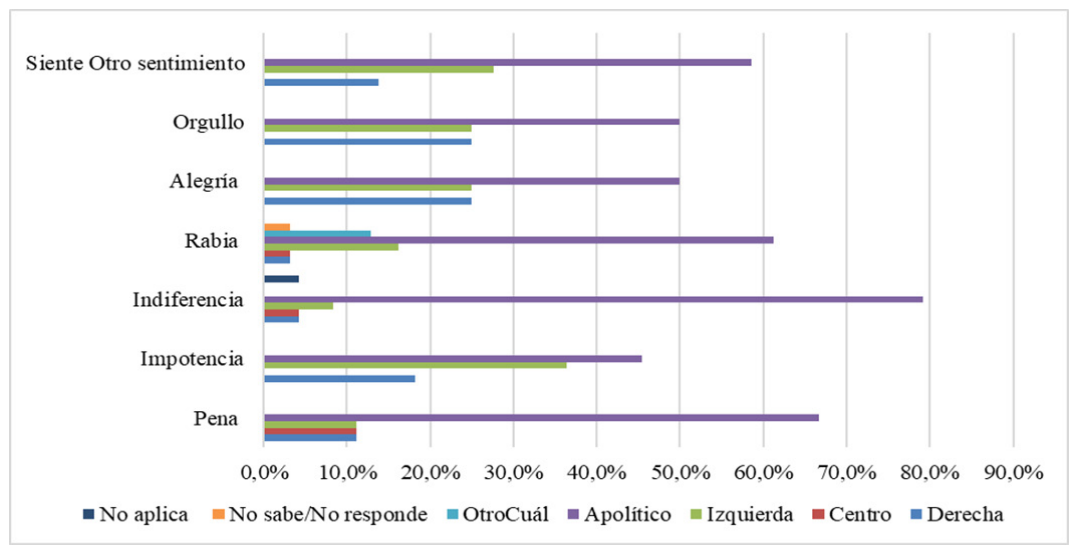

Fuente: Encuesta realizada para el curso Taller de Investigación-Sondeo

(primer semestre académico de 2015). Elaboración propia. 


\section{Dimensión No. 2: percepción de los hechos}

Ala pregunta deúnica respuesta sobre laviolencia de la toma, del total de personas que respondieron que para ellos había sido muy violento este suceso, el 23,7\% tenía de 60 años en adelante y el 20,3\% entre 53 y 59 años; más de la mitad, es decir, 62,7\% se denominan apolíticos y el 20,3\% de izquierda. Del total de personas que respondieron que para ellos había sido relativamente violento este suceso, el 31,3\% tenía entre 53 a 59 años y el 25\% entre 32 a 38 años; la mayoría es decir el $75 \%$ se denominaban apolíticos y el $18,8 \%$ de derecha. Del total de personas que respondieron que para ellos había sido poco violento este suceso, el 20\% tenía entre 32 a 38 años, el 20\% entre 39 a 45 años, el 20\% tenía entre 46 a 52 años y el 20\% 60 años en adelante; la mayoría, es decir un 60\% se denominaban apolíticos, el 30\% pertenecientes a un partido de izquierda y el 10\% a un partido de derecha. Del total de personas que respondieron que para ellos no había sido violento este suceso, el 100\% tenía de 39 a 45 años; la mitad 50\% se denominaba como apolítico y la otra mitad 50\% se denominaba de izquierda. Cabe resaltar que de las personas que contestaron "No sabe/No" responde respecto al carácter violento de la toma militar, el 27,8\% tenía de 25 a 31 años y el 22,2\% de 60 años en adelante; la mayoría, es decir el $55,6 \%$ se consideraban apolíticos, el 22,2\% de derecha y el 11,4\% de izquierda. (Ver Gráfico No. 5 y Gráfico No. 6).

Respecto a lo descrito anteriormente, cabe resaltar la percepción que se tiene respecto al nivel de violencia que tuvo la toma militar: para quienes vivenciaron aquella época, sobresale la opción Relativamente violenta y Muy violenta, mientras que generaciones posteriores consideran el hecho como poco violento (Ver Gráfica No. 5). Es así como sobre lo ocurrido en Siloé, se evidencia un mayor impacto en la memoria colectiva que tienen las personas que estuvieron cercanas -cronológicamente hablando- al hecho, mientras que a medida que transcurre el tiempo, esa huella del impacto de violencia se ha ido atenuando en las nuevas generaciones ya que no fueron directamente 
ellos quienes vivenciaron y experimentaron las consecuencias de lo ocurrido y conocen hasta cierto punto la historia por lo que a ellos se les ha comunicado.

Gráfico No. 5: ¿Qué tan violenta cree que fue la toma?

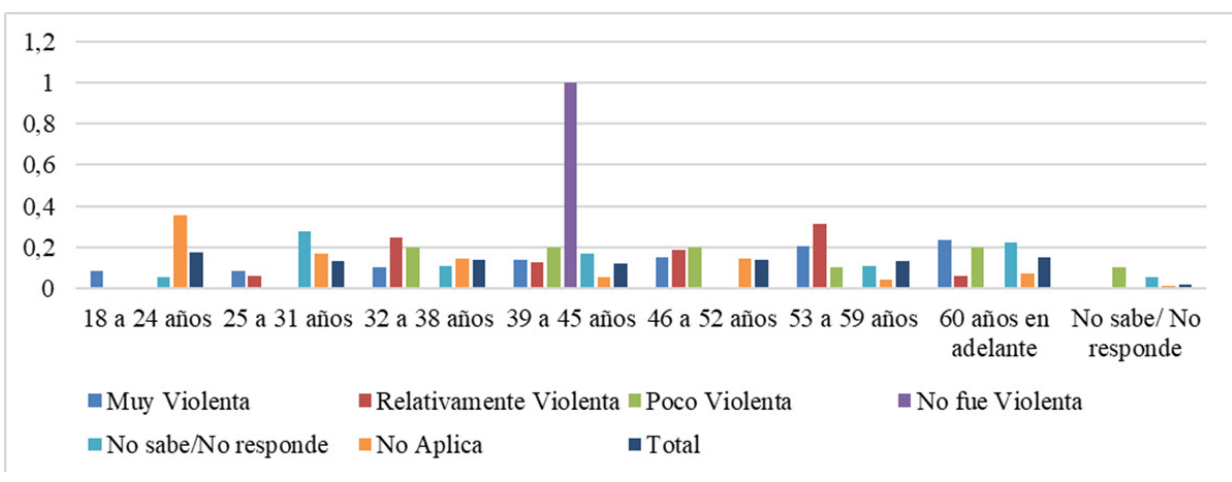

Fuente: Encuesta realizada para el curso Taller de Investigación-Sondeo

(primer semestre académico de 2015). Elaboración propia.

Gráfico No. 6: ¿Qué tan violenta cree que fue la toma?

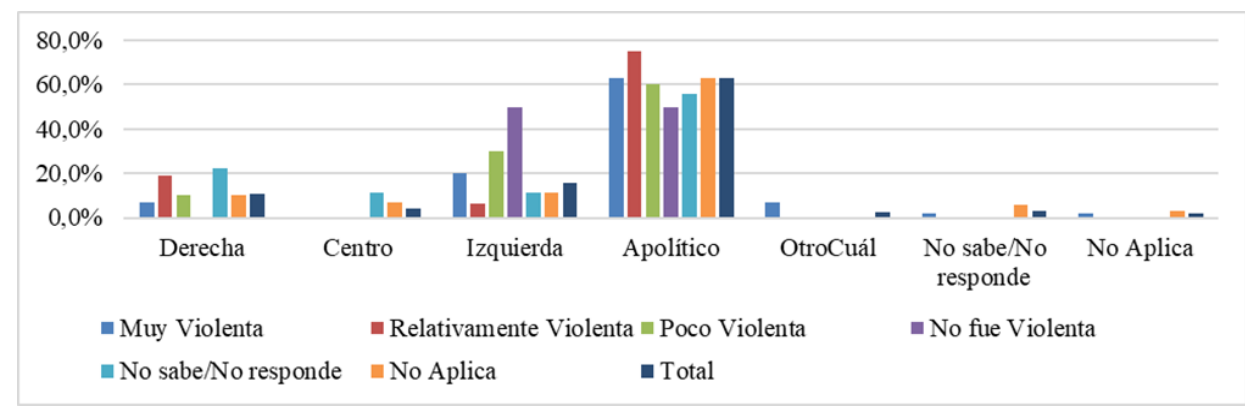

Fuente: Encuesta realizada para el curso Taller de Investigación-Sondeo

(primer semestre académico de 2015). Elaboración propia. 
En este punto se puede evidenciar un consenso relativamente amplio acerca del carácter violento de los hechos de la Toma (Ver Grafica No.5 y Gráfica N.6), sin embargo, la interpretación de los efectos que los actos violentos tuvieron en la convivencia y la seguridad del barrio, se encuentran polarizados entre positivos y negativos. Lo que cabe resaltar de este resultado en términos de memoria colectiva y su relación con la identidad social, es que en torno a este aspecto se produce una polarización del recuerdo que no corresponde con un acuerdo público ni una idea común prestablecida (Halbwachs, 2002).

\section{Dimensión No. 3: percepción acerca de las causas del hecho}

A la pregunta de única respuesta sobre si el M-19 tenía una presencia en el barrio al momento de la toma, del total de personas que respondieronafirmativamente, el 19,8\% tenía de 53 a 59 años y el 17,3\% de 60 años en adelante; la mayoría, es decir un 60,5\% se denominaba como apolítico. Del total de personas que respondieron que no creían que este grupo guerrillero tuviera una presencia en el barrio, el 40\% tenía de 53 a 59 años; el 40\% se denominaba como apolítico y el 40\% de un partido de izquierda (Ver Gráfico No. 7 y Gráfico No. 8).

En esta pregunta se evidencia de qué forma la memoria colectiva no es siempre fiel a lo realmente ocurrido, debido a que su constitución está influenciada por diversos factores que hacen que cada individuo interiorice los hechos de acuerdo a su propia subjetividad (Halbwachs, 2002). Es por este motivo que ante una pregunta como lo es la presencia del grupo insurgente M19 en el barrio en el momento de la toma, conociéndose de ante mano la respuesta afirmativa a este cuestionamiento, no resulta sorprendente que los habitantes que estuvieron en el mismo espacio cronológico en que sucedieron los hechos, aseguraran de forma opuesta a lo ocurrido, que no había presencia de ese grupo en ese entonces, o en otro caso, "No sabe/ 
no responde" con significativa identidad apolítica. Existen diversas explicaciones a esto, como el desconocimiento del contexto social en el momento que el hecho ocurrió, o el miedo por no querer involucrarse en ninguna postura determinista que pueda traer perjuicios a su integridad, entre otros; no obstante, cada factor que hace posible que la versión real de los hechos se tergiverse en relación con la que se narra, tiene no sólo una influencia social sino también subjetiva que genera diversas perspectivas y maneras de contemplar un mismo hecho (Halbwachs, 1991).

Gráfico No. 7: Al momento de la toma, ¿Había presencia del M-19 en el barrio?

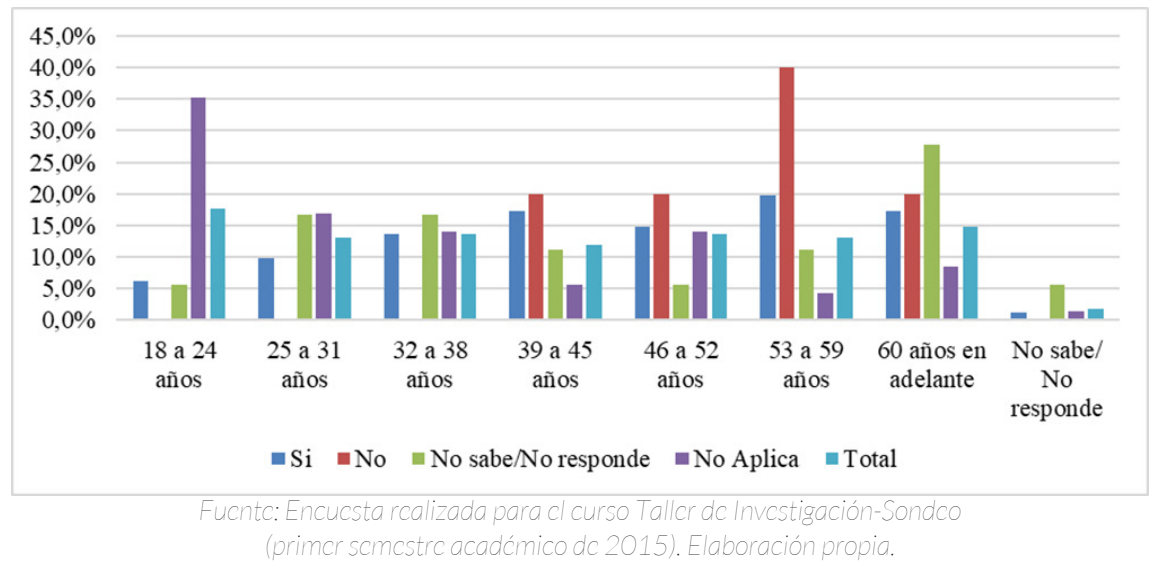

Gráfico No. 8: Al momento de la toma, ¿Había presencia del M-19 en el barrio?

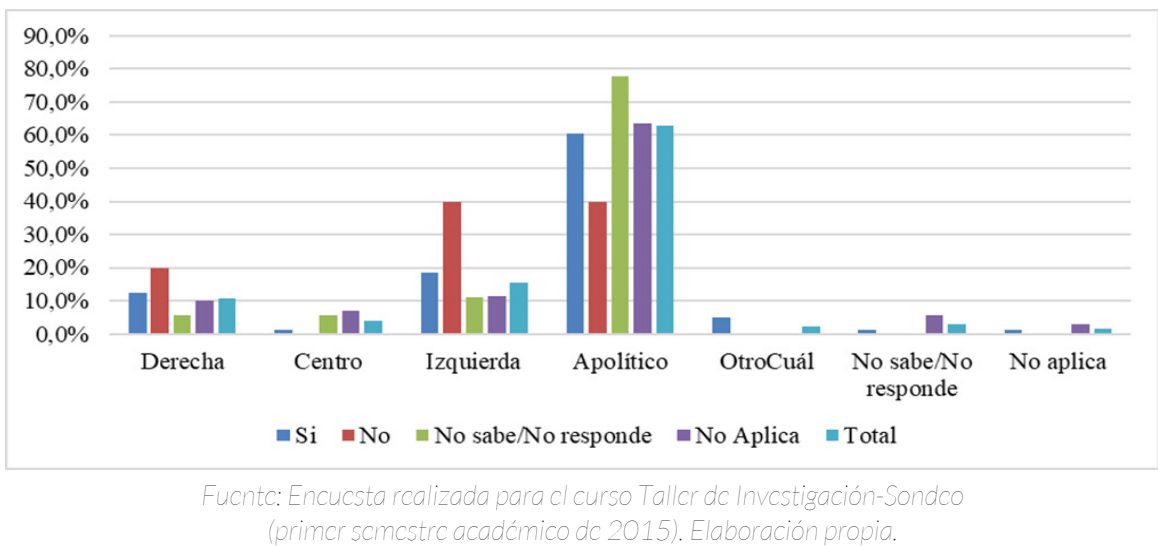


A la pregunta de múltiple respuesta sobre a quiénes se consideraba que iba dirigida la toma, del total de personas que respondieron que iba dirigida a la población civil, el 27,3\% tenía entre 46 a 52 años; la mayoría, es decir un 90,9\% se denominaba como apolíticos. Del total de personas que respondieron que la toma iba dirigida a las bandas criminales y/o pandillas, el 28,6\% tenía entre 39 a 45 años y el 21,4\% de 32 a 38 años; el 35,7\% se denominaba como apolítico y el 35,7\% de un partido de derecha. Del total de personas que respondieron que la toma iba dirigida a los guerrilleros del M-19, el 21,1\% tenía de 60 años en adelante y el 19,7\% de 53 a 59 años; la mayoría es decir el $62 \%$ se denominaba como apolítico y el $21,1 \%$ de un partido político de izquierda. Del total de personas que respondieron que la toma iba dirigida a "otros actores", el 50\% tenía de 46 a 52 años y el 50\% de 53 a 59 años; el $50 \%$ se denominada de un partido político de izquierda (Ver Gráfico No. 9 y Gráfico No. 10).

Con base a lo anteriormente expuesto, cabe resaltar que, al indagar sobre a quién se cree que iba dirigido el acto, las respuestas predominantes arrojan dos resultados: La población civil y el M-19. Esta respuesta se considera relevante, pues pone en evidencia una postura menos intermedia en comparación con otras preguntas, pues por lo general la población de 60 años en adelante tiende a optar por la opción "No sabe/No responde" como respuesta, mientras que en este caso es clara la forma como los encuestados de este rango generacional leen a los actores que fueron víctimas de este suceso. En este caso, los habitantes de Siloé no sólo manifiestan reconocer contra quien se efectuó el golpe, sino que además se autodenominan afectados al ser parte de esa población civil (Páez et al., 1998). 
Gráfica No. 9: ¿La toma iba dirigida a...?

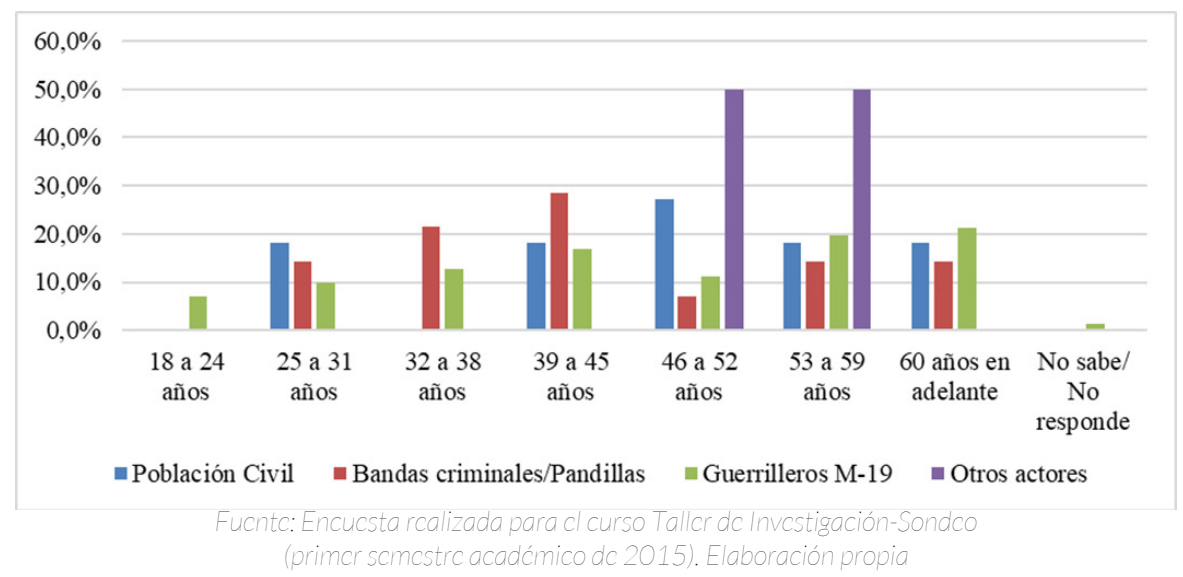

Gráfico No. 10: ¿La toma iba dirigida a...?

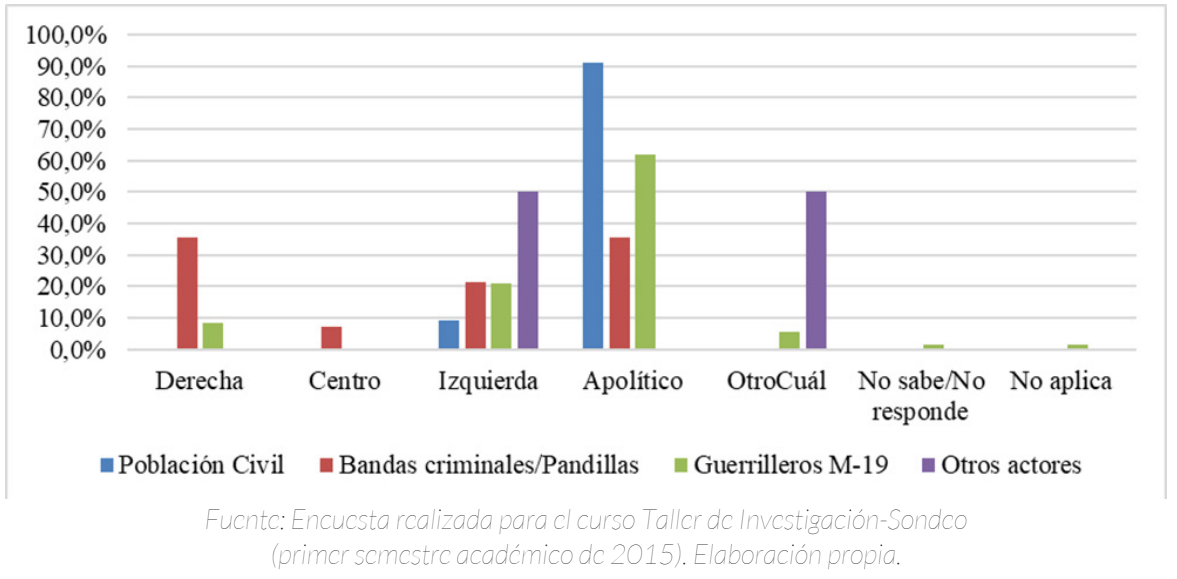

\section{Dimensión No. 4: proyección futura}

En la pregunta de respuesta múltiple sobre cómo considera que serían las condiciones económicas en el barrio de no haberse dado la toma, del total de personas que respondieron "mejor económicamente", el 22,2\% tenía de 60 años en adelante; la mayoría, es decir un 63\% se denominaba como apolítico. Del total de personas que respondieron 
"igual económicamente", el 22,2\% tenía 60 años en adelante; la mayoría, es decir un 60,5\% se denominaba como apolítico. Del total de personas que respondieron "peor económicamente", el 25\% tenía entre 46 a 52 años y el 25\% tenía 60 años en adelante; el 50\% se denominaba como apolítico, el 16,7\% de izquierda y el 8,3\% de derecha (Ver Gráfico No. 11 y Gráfico No. 12).

Gráfico No. 11: ¿Cómo serían las condiciones económicas en el barrio de no haberse dado la toma?

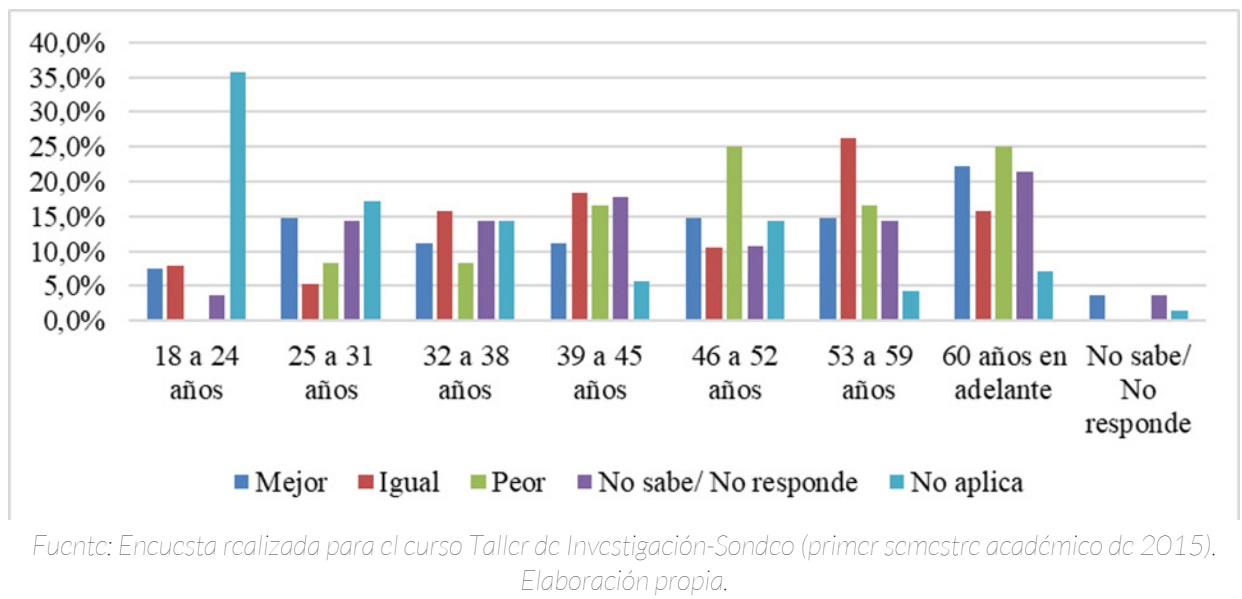

Gráfico No. 12: ¿Cómo serían las condiciones económicas en el barrio de no haberse dado la toma?

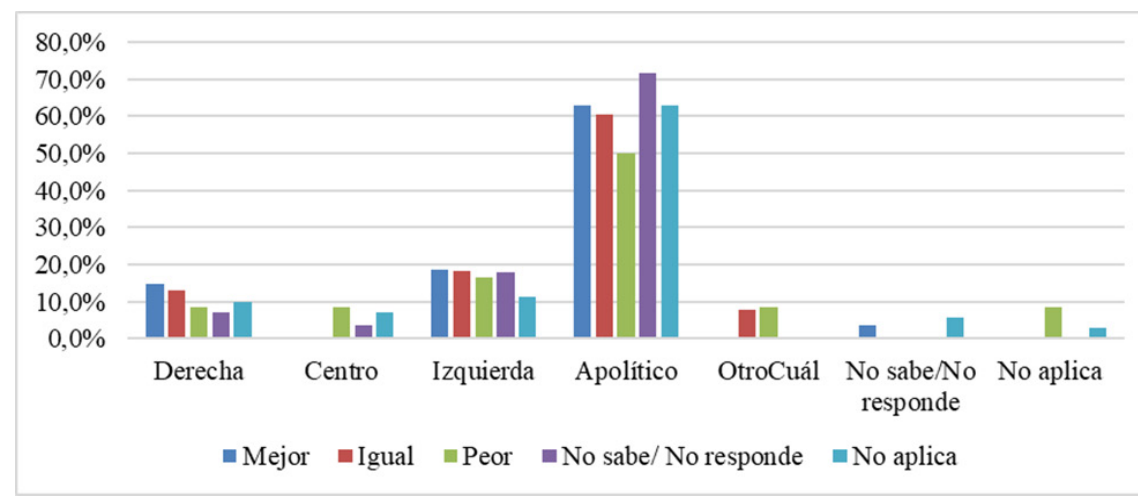

Fuente: Encuesta realizada para el curso Taller de Investigación-Sondeo

(primer semestre académico de 2015). Elaboración propia. 
En la pregunta sobre cómo considera que serían las condiciones de seguridad en el barrio de no haberse dado la toma, del total de personas que respondieron "mejor en seguridad", el 23,7\% tenía entre 39 y 45 años; la mayoría, es decir un $57,9 \%$ se denominaba como apolítico. Del total de personas que respondieron "igual en seguridad", el 33,3\% tenía entre 53 y 59 años; la mayoría, es decir un 56,7\% se denominaba como apolítico. Del total de personas que respondieron "peor en seguridad", el 40\% tenía entre 46 y 52 años; la mayoría, es decir un 66,7\% se denominaba como apolítico (Ver Gráfico No. 13 y Gráfico No. 14).

Gráfico No.13: ¿Cómo serían las condiciones de seguridad en el barrio de no haberse dado la toma?

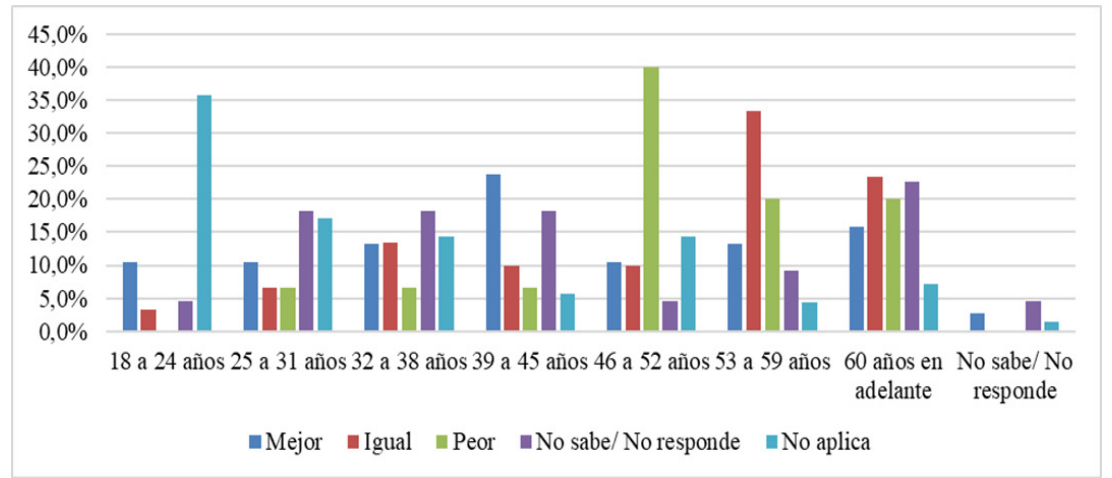

Fuente: Encuesta realizada para el curso Taller de Investigación-Sondeo

(primer semestre académico de 2015). Elaboración propia.

Gráfica No. 14: ¿Cómo serían las condiciones de seguridad en el barrio de no haberse dado la toma?

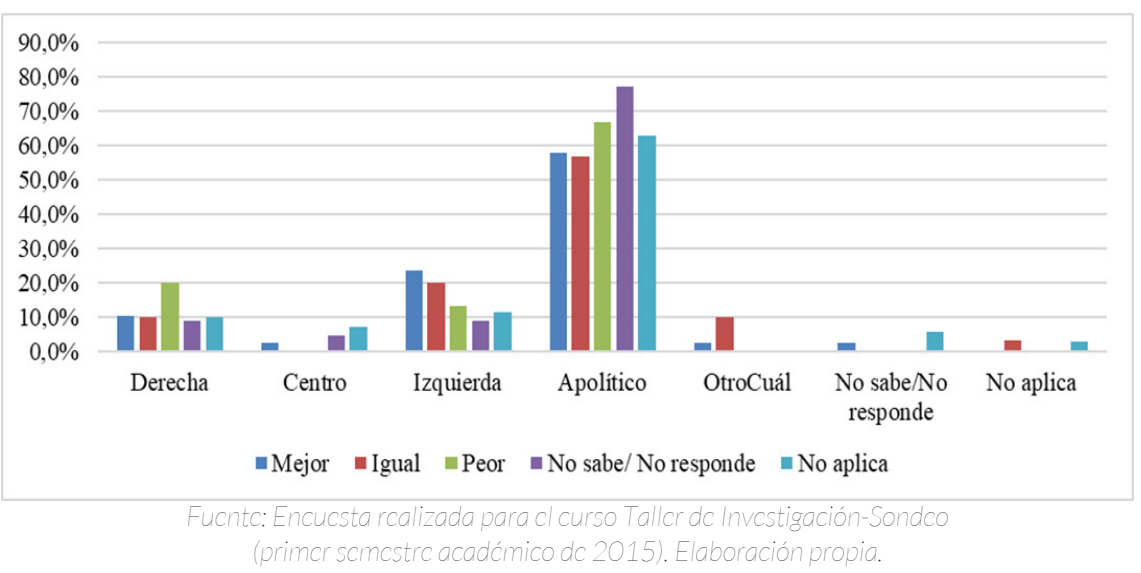


En la pregunta sobre cómo considera que serían las condiciones de convivencia en el barrio de no haberse dado la toma, del total de personas que respondieron "mejor en convivencia”, el 27\% tenía entre 53 y 59 años; la mayoría, es decir un 51,4\% se denominaba como apolítico. Del total de personas que respondieron "igual en convivencia", el 17,9\% tenía entre 32 y 38 años, el 17,9\% tenía entre 18 a 24 años y el 17,9\% tenía entre 46 a 52 años; la mayoría, es decir un 67,9\% se denominaba como apolítico. Del total de personas que respondieron "peor en convivencia", el 28,6\% tenía entre 53 a 59 años; la mayoría, es decir un 64,3\% se denominaba como apolítico (Ver Gráfica No. 15 y Gráfica No. 16)

Gráfica No. 15: ¿Cómo serían las condiciones de convivencia en el barrio de no haberse dado la toma?

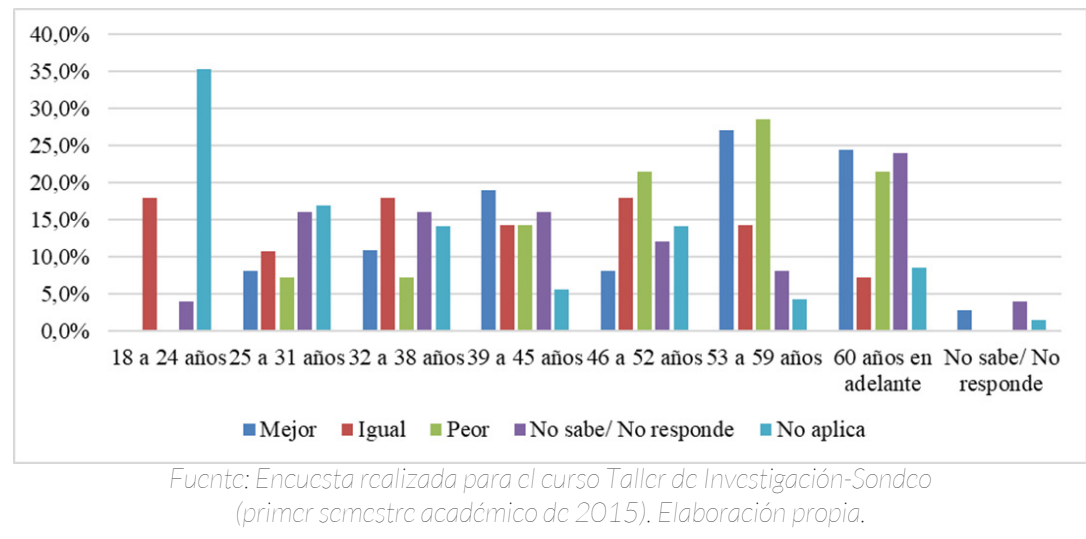

Gráfica No. 16: ¿Cómo serían las condiciones de convivencia en el barrio de no haberse dado la toma?

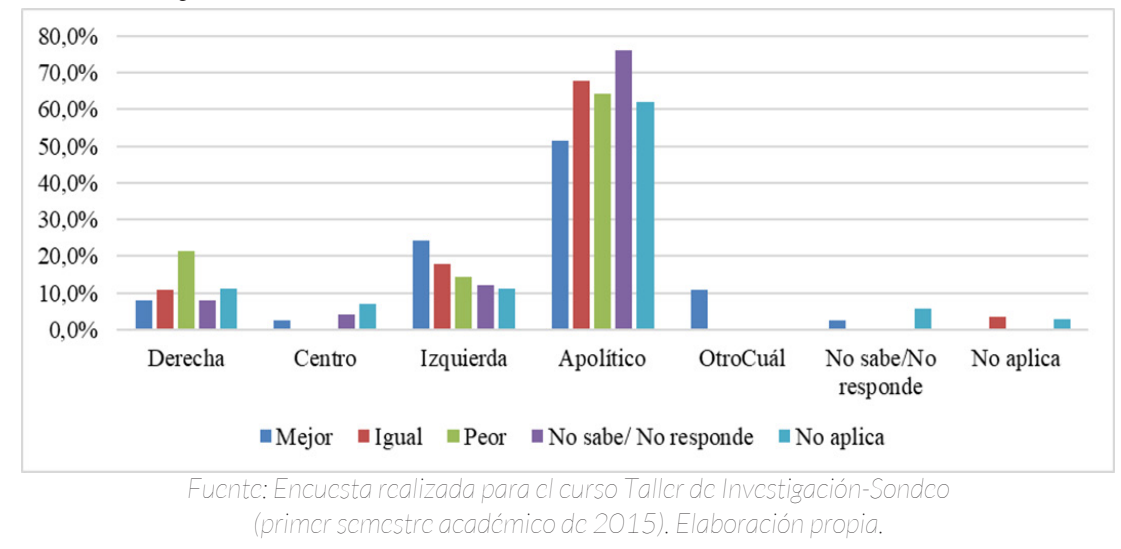


En este punto, se puede evidenciar (ver Gráfica No. 11, Gráfica No. 12, Gráfica No. 13, Gráfica No. 14, Gráfica No. 15, Gráfica No.16) un consenso relativamente amplio acerca del carácter violento de los hechos de la Toma, sin embargo, la interpretación de los efectos que los actos violentos tuvieron en la convivencia y la seguridad del barrio, se encuentran polarizados entre positivos y negativos. Lo que cabe resaltar de este resultado en términos de memoria colectiva y su relación con la identidad social, es que en torno a este aspecto se produce una polarización del recuerdo que no corresponde con un acuerdo público ni una idea común prestablecida (Halbwachs, 1991).

\section{Conclusiones}

Como parte de la reflexión final, se puede afirmar que la memoria que se hace respecto a lo ocurrido el 1 de diciembre de 1985, para los habitantes del barrio Siloé, se cristaliza de manera compleja, pues en el ejercicio de recordar se combinan múltiples interpretaciones de lo ocurrido y así mismo, distintas maneras de asimilar e interiorizar sus efectos en el aspecto personal, la forma de relacionarse con la política y su vida cotidiana en general.

Corolario a lo anterior, se puede afirmar que la condición sociodemográfica de rango generacional y la identidad política, están vinculadas con la construcción de memoria colectiva en la comuna 20 de Cali, más puntualmente en los barrios Tierra Blanca, Lleras Camargo y Pueblo Joven, respecto a la toma militar. Sin embargo, se debe aclarar que en la mayoría de las respuestas las variables como el sexo (femenino) y el lugar de residencia (Tierra Blanca y Lleras Camargo) son más significativos, y, por ende, esto influye notablemente en los resultados. Es por este motivo que la variable de edad o rango generacional fue la que permitió la comparación de los efectos del hecho paracada sector de la población, de acuerdo con la cercanía que tuvieron a lo ocurrido en el 
plano cronológico, para así corroborar el valor que la memoria colectiva tiene en cómo se construyen los recuerdos y su influencia en el plano personal. Además, vale la pena resaltar que en un contexto social tan cambiante como lo es el colombiano, una divergencia de edad de una o más décadas, hace la diferencia debido a los múltiples acontecimientos políticos, militares y sociales que cada época conlleva en el país. Por esta razón, a pesar de que existen grupos de edad muy cercanos como de 46 a 52, de 53 a 59 y de 60 años en adelante, en muchos casos se encontraron diferencias significativas en sus respuestas siendo en algunos casos completamente opuestas.

Asimismo, el recuerdo de estos hechos suscita una polarización dependiendo de la edad de los encuestados. En algunas ocasiones, bien sea porque no han tenido una experiencia directa con el hecho y están influenciados por la posición que otros tengan al respecto, o también, en el caso de quienes lo vivieron, por el contexto previo o posterior al hecho, lo cual lleva a que se generen sesgos de lo que se vivió o la posición que se cree se debe asumir frente a ello para evitar inconvenientes de cualquier tipo.

También, cabe resaltar que las personas encuestadas que se autodenominan sin ninguna orientación política -apolíticos- resultan una población especialmente interesante, pues se han constituido como uno de los sectores más significativo y numeroso. Su perfil es, en general, consistente con el de un grupo que muestra desafección y rechazo hacia el sistema político, manifestando menor interés y conocimiento acerca de asuntos con connotaciones ideológicas. Los resultados muestran que este grupo se ha distanciado de posturas radicales sobre lo ocurrido, prefiriendo ubicarse en una actitud en la que optan por la opción "No sabe/no responde" para dar respuesta a preguntas que implican asumir una postura determinada. 


\section{Bibliografía}

Alonso, J. C., Solano, M. A., Vera, R., \& Gallego, A. I. (2007). Una mirada descriptiva a las comunas de Cali. Cali: Cienfi Universidad Icesi.

Betancourt Delgado, C., \& Molina Orrego, M. F. (2014). La música como vehículo de transformación social en niños y jóvenes dentro del proyecto tambores de Siloé de la comuna 20 de la ciudad de Cali.

Caldono Ávila, C. O., Echeverry Gaviria, P. A., \& Pacheco Espinoza, D. M. (2012). Siloé no es como la pintan. Cali: Universidad del Valle.

Departamento Administrativo de Gestión del Medio Ambiente -DAGMA. COMUNA 20. PANORAMA AMBIENTAL [En línea]. [Consultado el 16 de mayo de 2015]. Disponible en internet: http:// www.cali.gov.co/publico2/gobierno/dagmaweb/comuna20.htm\#A.

Desde Abajo. (2011). Siloé: una colina de pueblo y agua. Desde Abajo: la otra posición para leer. [En línea]. Recuperado el 16 de mayo de 2015 de https://www.desdeabajo.info/ediciones/item/8803silo\%25C3\%25A9-una-colina-de-pueblo-y-agua.html

García Márquez, G. (2002). Vivir para contarla. New York: Alfred A. Knopf.

García, Rolando. (2013) Sistemas complejos. Concepto, método y fundamentación epistemológica de la investigación transdisciplinaria. México: GEDISA.

Halbwachs, M., \& Aguilar Díaz, M. Á. (2002). Fragmentos de la memoria colectiva. Athenea digital: revista de pensamiento e investigación social, (2), 103-113.

Manzi, J., Helsper, E., Ruiz, S., Krause, M., \& Kronmüller, E. (2003). El pasado que nos pesa: la memoria colectiva del 11 de septiembre de 1973.Revista de ciencia política (Santiago), 23(2), 177-214. 
Páez, D., Nekane B. y González, J. (1998). "Memoria Colectiva y Traumas Políticos: Investigación transcultural de los procesos sociales de recuerdo de sucesos traumáticos". En Memorias de procesos culturales y políticos, editado por D. Páez, J.F. Valencia, J.W. Pennebaker, B. Rime y D. Jodelet. Bilbao: Servicio Editorial Universidad del país Vasco 171206.

Ramos, Ramón. (1989). "Maurice Halbwachs y la memoria colectiva”. Revista de Occidente 100, 63 - 81.

Revista Semana. Consultado el 20 de junio del 2016, disponible en http://www.semana.com/nacion/articulo/guerra-total-guerrasucia/7261-3

Sears, David O. (1990). "Whither Political Socialization Research? The Question of Persistence". En Political Socialization, Citizenship Education, and Democracy, editado por O. Ichilov. New York: Teachers College Press, 69-97.

Steward, Abigail J. y Joseph M. Jr.Healy (1989). "Linking Individual Development and Social Changes". American Psychologist 44 (1), 3042.

Ulloa Sanmiguel, A. (2011). Cali, Capital deportiva, ciudad cívica y sede del narcotráfico: tres representaciones sociales urbanas. Revista Nexus Comunicación, (6).

Watts, Meredith W. (1999). "Are there Typical Age Curves in Political Behavior? The Age Invariance. Hypothesis and Political Socialization". Political Psychology 20 (3), 477-499 